International Journal of Pure and Applied Mathematics

Volume 84 No. 2 2013, 81-92

ISSN: 1311-8080 (printed version); ISSN: 1314-3395 (on-line version)

url: http://www.ijpam.eu

doi: http://dx.doi.org/10.12732/ijpam.v84i2.7

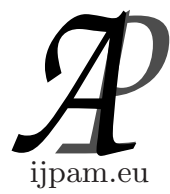

\title{
CONSTRUCT ASYMPTOTICALLY OPTIMAL CONTROL OF SYSTEMS IN CONDITIONALLY STABLE CASE
}

\author{
I. Boitsova \\ Odesa State Environmental University \\ Faculty of Computer science \\ Lvivs'ka st., 15, Odesa, 65016, UKRAINE
}

\begin{abstract}
This paper considers an optimal control problem, which is described by a system of differential equations with fast and slow variables and boundary conditions on fast variables. Conditions that guarantee construct of asymptotic approximation to a solution in the conditionally stable case, which is being used for an averaged optimal control problem for slow variables, are imposed upon a fast subsystem. The paper provides an algorithm, which allows to construct an optimal control of the given optimal control problem, by means of an optimal control of the averaged problem. Found solutions are also being assessed.
\end{abstract}

AMS Subject Classification: 34B15, 34C29, 34K35

Key Words: averaging method, boundary value problem, slow and fast variables, conditionally stable case, optimal control problem

\section{Introduction}

This paper considers the optimal control problem, which is described by a system of differential equations with fast and slow variables and with the boundary conditions on the fast variables. The fast subsystem conditions are imposed to ensure the construction of the asymptotic approximation to the solution in a

Received: January 24, 2013

(c) 2013 Academic Publications, Ltd. url: www.acadpubl.eu 
conditionally stable case, which is used for the construction of the average optimal control problem for the slow variables. The algorithm, which is on the optimal management of the homogenized problem can construct an asymptotically optimal control of a given optimal control problem. Provides estimates of the solutions.

\section{Asymptotic Solution of the Boundary Value Problem for System of Differential Equations in the Conditionally Stability Case}

The boundary value problem for the system of differential equations with fast and slow variables and with the boundary conditions on the fast variables:

$$
\begin{aligned}
& \dot{x}=\varepsilon f(t, x, y), \quad x(0)=x^{0}, \\
& \dot{y}=g(x, y), \quad R(y(0), y(T))=0,
\end{aligned}
$$

is considered. Here $t \in[0, T], T=L \varepsilon^{-1}, L>0, \varepsilon>0$ is a small parameter, $x \in D \subset R^{n}, \quad y \in Q \subset R^{m}$.

Let us consider the degenerate boundary value problem for problem (1), (2) as $\varepsilon=0$ :

$$
\begin{gathered}
\dot{x}=0, \quad x(0)=x^{0}, \\
\dot{y}=g(x, y), \quad R(y(0), y(T))=0 .
\end{gathered}
$$

Let $\tau=\varepsilon t$ be a slow time in problem (4). Ptoblem (4) is a singularly perturbed boundary value problem. The solution of this problem will be constract according to the algorithm given in [2] as

$$
h(t, x(t))=y_{0}(t, x(t))+\Pi y\left(t-0 \varepsilon^{-1}, x(0)\right)+Q y\left(t-L \varepsilon^{-1}, x(T)\right),
$$

where $y_{0}(t, x(t))$ is an isolated root of the nonlinear equation $g(x, y(t))=0$; $\Pi y\left(t-0 \varepsilon^{-1}, x(0)\right)$ is a boundary layer for rapid changes in the neighborhood of $t=0 \varepsilon^{-1}, Q y\left(t-L \varepsilon^{-1}, x(T)\right)$ is a boundary layer to rapid changes in the neighborhood of $t=L \varepsilon^{-1}$, where $x$ as a parameter.

Let for $y_{0}(t, x)$ as a solution of equation $g(x, y(t))=0$ the eigenvalues of the matrix

$$
\frac{\partial g\left(x, y_{0}(t, x)\right)}{\partial y}
$$

satisfies the next conditions

$$
\begin{aligned}
& \operatorname{Re} \lambda_{i}(t)<0, \quad i=\overline{1, m_{1}}, \quad m_{1}<m, \\
& \operatorname{Re} \lambda_{i}(t)>0, \quad i=\overline{m_{1}+1, m}, \quad t \in[0, T] .
\end{aligned}
$$




$$
\begin{aligned}
& \lambda_{i}(t) \neq \lambda_{j}(t), \quad i \neq j, \\
& \operatorname{Re} \lambda_{1}(t) \leq \operatorname{Re} \lambda_{2}(t) \leq \ldots \leq \operatorname{Re} \lambda_{m}(t), \quad t \in[0, T] .
\end{aligned}
$$

These conditions describe the solutions in conditionally stability case.

Let us compose the matrix $B(t)$ as the set columns linearly independent eigenvectors of the matrix (6). The bloks $B_{11}(t)$ and $B_{22}(t)$ of matrix $B(t)$ correspond eigenvalues with negative and positive real parts and following conditions hold:

$$
\begin{array}{r}
\operatorname{det} B_{11}\left(0 \varepsilon^{-1}\right) \neq 0, \quad \operatorname{det} B_{22}\left(0 \varepsilon^{-1}\right) \neq 0, \\
\operatorname{det} B_{11}\left(L \varepsilon^{-1}\right) \neq 0, \quad \operatorname{det} B_{22}\left(L \varepsilon^{-1}\right) \neq 0 .
\end{array}
$$

An asymptotic approximation of the solution of the problem (4) has two boundary layers in this case. Both layers can be determined from next conditions:

$$
\begin{array}{r}
\frac{d \Pi y\left(t_{1}, x(0)\right)}{d t_{1}}=g\left(x(0), y_{0}(0, x(0))+\Pi y\left(t_{1}, x(0)\right)\right), \\
\frac{d Q y\left(t_{2}, x(T)\right)}{d t_{2}}=g\left(x(T), y_{0}(T, x(T))+Q y\left(t_{2}, x(T)\right)\right), \\
R\left(y_{0}(0, x(0))+\Pi y(0, x(0)), y_{0}(T, x(T))+Q y(0, x(T))\right)=0,
\end{array}
$$

where

$$
t_{1}=t-0 \varepsilon^{-1}, \quad t \geq 0 \varepsilon^{-1}, \quad t_{2}=t-L \varepsilon^{-1}, \quad t \leq L \varepsilon^{-1} .
$$

The solution (5) for fast variables substitute into problem for slow variables (1) and then we obtain the perturbed problem:

$$
\dot{\tilde{x}}=\varepsilon f(t, \tilde{x}(t), h(t, \tilde{x}(t))), \quad \tilde{x}(0)=x^{0} .
$$

If into the problem (1) substitute the function $y_{0}(t, x)$ as an isolated root of nonlinear equations $g(x, y(t))=0$ we obtain the partial averaging problem:

$$
\dot{\bar{x}}=\varepsilon f\left(t, \bar{x}(t), y_{0}(t, \bar{x}(t))\right), \quad \bar{x}(0)=x^{0} .
$$

Let consider a curve $L_{0}$ as set of three curves $L_{01}, L_{02}, L_{03}$ :

$$
\begin{gathered}
L_{01}=\left\{(x, y, t): x=\bar{x}(0), y=y_{0}(0, \bar{x}(0))+\Pi y\left(t_{1}, \bar{x}(0)\right), t_{1} \geq 0, t=0\right\}, \\
L_{02}=\left\{(x, y, t): x=\bar{x}(t), y=y_{0}(t, \bar{x}(t)), 0 \leq t \leq T\right\}, \\
L_{03}=\left\{(x, y, t): x=\bar{x}(T), y=y_{0}(T, \bar{x}(T))+Q y\left(t_{2}, \bar{x}(T)\right), t_{2} \leq 0, t=T\right\} .
\end{gathered}
$$

Theorem 1. Suppose the following conditions hold:

I) Function $g(x, y)$ is twice continuously differentiable in $\delta$-neighborhood of curve $L_{0}$; 
II) Equation $g(x, y)=0$ has an isolated root $y=y_{0}(t, x)$ in closed bounded region of space variables, the function $y_{0}(t, x)$ is continuous in the region and satisfies the Lipschitz condition by $x$ with constant $H>0$;

III) Problem (14) has an unique solution as $t \in[0 ; T]$ corresponding to the root $y_{0}(t, x)$; the function $f\left(t, x, y_{0}(t, x)\right)$ is continuous in a $\delta$-neighborhood of the curve $L_{0}$ and satisfies a Lipschitz condition by $x$ and $y$ with constant $H>0$;

$I V)$ The eigenvalues $\lambda_{i}(\tau)$ of (6) satisfy (7), (8);

V) The conditions (9), (10) satisfaed.

The system

$$
\frac{d \gamma}{d t_{1}}=g\left(x(0), \quad \gamma+y_{0}(0, x(0))\right)
$$

has an integral manifold $S^{+}$. This manifold consists of trajectories of the system, which tend to the origin as $t_{1} \rightarrow+\infty$. Also this manifold can be represented as $\xi_{2}=\Phi_{2}\left(\xi_{1}\right)$, where $\xi_{1} \in \Omega^{+}$, the block $\xi_{1}$ contains $m_{1}$ components, the block $\xi_{2}$ has $\left(m-m_{1}\right)$ components of vector $\xi$.

The system

$$
\frac{d \eta}{d t_{2}}=g\left(x(T), \quad \eta+y_{0}(T, x(T))\right)
$$

has an integral manifold $S^{-}$. This manifold consists of trajectories of the system, which tend to the origin as $t \rightarrow-\infty$. Also this manifold can be represented as $\xi_{1}=\Phi_{1}\left(\xi_{2}\right)$, where $\xi_{2} \in \Omega^{-}$.

VI) Functional determinant $\frac{\partial R}{\partial y_{0}}$, built for the boundary condition is not zero: $\operatorname{det}\left(\frac{\partial R}{\partial y_{0}}\right) \neq 0, y_{01} \in \Omega^{+}, y_{02} \in \Omega^{-}$.

Then for all $\eta>0$ and for all $L>0$ exist the constants $\varepsilon_{0}(\eta, L)>0$ and $C>0$ such that as $\varepsilon \in\left(0, \varepsilon_{0}\right]$ in a $\delta$-neighborhood of the curve $L_{0}$ exists an unique solution of (1), (2) and as $t \in\left[0, L \varepsilon^{-1}\right]$ the following estimates hold:

$$
\begin{gathered}
\|x(t)-\tilde{x}(t)\| \leq C \varepsilon . \\
\|x(t)-\bar{x}(t)\| \leq \eta .
\end{gathered}
$$

Proof. First we will get the estimate (15). Let $x(t), y(t)$ be solutions of the problem $(1),(2)$ and $\tilde{x}(t)$ be a solution of the perturbed problem (13) as solution (5) of the degenerate problem (4).

It follows from the conditions of the theorem and from Theorem 4.2 [2] that there exists $\varepsilon_{1}>0, \quad C_{1}>0$ such that as $\varepsilon \in\left(0, \varepsilon_{1}\right]$ the asymptotic solution 
(5) of problem (4) in the zero-approximation satisfy the next inequality

$$
\|y(t, x)-h(t, x)\| \leq C_{1} \varepsilon
$$

Then

$$
\begin{gathered}
\|x(t)-\tilde{x}(t)\|=\varepsilon \cdot\left\|\int_{0}^{t} f(\tau, x(\tau), y(\tau)) d \tau-\int_{0}^{t} f(\tau, \tilde{x}(\tau), h(\tau, \tilde{x}(\tau))) d \tau\right\| \leq \\
\leq \varepsilon \int_{0}^{t}\|f(\tau, x(\tau), y(\tau))-f(\tau, x(\tau), h(\tau, x(\tau)))\| d \tau+ \\
+\varepsilon \int_{0}^{t}\|f(\tau, x(\tau), h(\tau, x(\tau)))-f(\tau, \tilde{x}(\tau), h(\tau, \tilde{x}(\tau)))\| d \tau \leq \\
\quad \leq \varepsilon H \int_{0}^{t}\|y(\tau, x(\tau))-h(\tau, x(\tau))\| d \tau+ \\
+\varepsilon H \int_{0}^{t}(\|x(\tau)-\tilde{x}(\tau)\|+\|h(\tau, x(\tau))-h(\tau, \tilde{x}(\tau))\|) d \tau \leq \\
\leq \varepsilon H C_{1} L+\varepsilon H(1+H) \int_{0}^{t}\|x(\tau)-\tilde{x}(\tau)\| d \tau .
\end{gathered}
$$

By Gronwall-Bellman lemma we have

$$
\|x(t)-\tilde{x}(t)\| \leq \varepsilon H C_{1} L e^{H(1+H) L},
$$

therefore, estimate $(15)$ as $C=H C_{1} L e^{H(1+H) L}$.

Now we will get the estimate $(16)$. Let $\bar{x}(t)$ be the solution of partially averaged problem $(14)$ as $y_{0}(t, x)$ as a solution of equation $g(x, y(t))=0$.

Then

$$
\|x(t)-\bar{x}(t)\| \leq\|x(t)-\tilde{x}(t)\|+\|\tilde{x}(t)-\bar{x}(t)\| \leq C \varepsilon+\|\tilde{x}(t)-\bar{x}(t)\| .
$$

Now we will estimate the follow difference

$$
\|\tilde{x}(t)-\bar{x}(t)\|=
$$




$$
\begin{aligned}
& =\varepsilon \cdot\left\|\int_{0}^{t} f(\tau, \tilde{x}(\tau), h(\tau, \tilde{x}(\tau))) d \tau-\int_{0}^{t} f\left(\tau, \bar{x}(\tau), y_{0}(\tau, \bar{x}(\tau))\right) d \tau\right\| \leq \\
& \leq \varepsilon \int_{0}^{t}\|f(\tau, \tilde{x}(\tau), h(\tau, \tilde{x}(\tau)))-f(\tau, \bar{x}(\tau), h(\tau, \bar{x}(\tau)))\| d \tau+ \\
& +\varepsilon \int_{0}^{t}\left\|f(\tau, \bar{x}(\tau), h(\tau, \bar{x}(\tau)))-f\left(\tau, \bar{x}(\tau), y_{0}(\tau, \bar{x}(\tau))\right)\right\| d \tau \leq \\
& \leq \varepsilon H \int_{0}^{t}(\|\tilde{x}(\tau)-\bar{x}(\tau)\|+\|h(\tau, \tilde{x}(\tau))-h(\tau, \bar{x}(\tau))\|) d \tau+ \\
& +\varepsilon H \int_{0}^{t}\left\|h(\tau, \bar{x}(\tau))-y_{0}(\tau, \bar{x}(\tau))\right\| d \tau \leq \\
& \leq \varepsilon H(1+H) \int_{0}^{t}\|\tilde{x}(\tau)-\bar{x}(\tau)\| d \tau+ \\
& +\varepsilon H \int_{0}^{t}\left\|\Pi y\left(t-0 \varepsilon^{-1}, \bar{x}(0)\right)\right\| d \tau+\varepsilon H \int_{0}^{t}\left\|Q y\left(t-L \varepsilon^{-1}, \bar{x}(T)\right)\right\| d \tau .
\end{aligned}
$$

It follows from proof of theorem 4.2 in [2] that for the boundary functions in the asymptotic solution (5) of the degenerate problem (4) the next estimates are hold

$$
\begin{gathered}
\left\|\Pi y\left(t_{1}, x\right)\right\| \leq C_{2} e^{-s t_{1}}, \quad t_{1}=\frac{\tau-0}{\varepsilon} \geq 0, \\
\left\|Q y\left(t_{2}, x\right)\right\| \leq C_{2} e^{s t_{2}}, \quad t_{2}=\frac{\tau-L}{\varepsilon} \leq 0
\end{gathered}
$$

where $C_{2}$ and $s$ are constants. And for any $\eta_{1}>0$ there exist time moments $t_{1}^{*}=\frac{\tau-0}{\varepsilon^{*}}>0$ and $t_{2}^{*}=\frac{\tau-L}{\varepsilon^{*}}>0$ such that for all $t_{1}=\frac{\tau-0}{\varepsilon} \geq \frac{\tau-0}{\varepsilon^{*}}$ and $t_{2}=\frac{\tau-L}{\varepsilon} \leq \frac{\tau-L}{\varepsilon^{*}}$, that is why for all $\varepsilon \in\left(0, \varepsilon^{*}\right]$ the following inequalitys hold:

$$
\left\|\Pi y\left(t_{1}, x\right)\right\| \leq \eta_{1}, \quad\left\|Q y\left(t_{2}, x\right)\right\| \leq \eta_{1}
$$

Since (19) and (20) we obtain

$$
\|\tilde{x}(t)-\bar{x}(t)\| \leq \varepsilon H(1+H) \int_{0}^{t}\|\tilde{x}(\tau)-\bar{x}(\tau)\| d \tau+2 H \eta_{1} L .
$$


By Gronwall-Bellman lemma we have

$$
\|\tilde{x}(t)-\bar{x}(t)\| \leq 2 H \eta_{1} L e^{H(1+H) L}=\eta_{2} .
$$

Using the last estimate and inequality (18) we have that for all $\eta>0$ and $L>0$ there exists $\varepsilon_{0}(\eta, L)=\min \left\{\varepsilon_{1}, \varepsilon^{*}\right\}>0$ such that for all $\varepsilon \in\left(0, \varepsilon_{0}\right]$ the estimate (16) is hold. The theorem is proved.

\section{Construction of Asymptotic Optimal Control of System in the Conditionally Stability Case}

Let us consider optimal control problem with boundary conditions on the fast variables

$$
\begin{gathered}
\dot{x}=\varepsilon[f(t, x, y)+A(x) \varphi(t, u)], \quad x(0)=x^{0}, \\
\dot{y}=g(x, y), \quad R(y(0), y(T))=0,
\end{gathered}
$$

and terminal criterion:

$$
J[u]=\Phi(x(T)) \rightarrow \min ,
$$

where $t \in[0, T], T=L \varepsilon^{-1}, L>0, \varepsilon>0$ is a small parameter, $x \in D \subset R^{n}$, $y \in Q \subset R^{m}, u(t) \in U \subset \operatorname{comp}\left(R^{r}\right), \operatorname{comp}\left(R^{r}\right)$ is a space of compact subset in $R^{r}$ with Hausdorff metric, $A(x)$ is $n \times p$-matrix.

Suppose that the problem (22) for the fast variables satisfies the conditions of construction of asymptotic approximation to the solution in a conditionally stability case and suppose that there exists an isolated solution $y_{0}(t, x)$ of the nonlinear equation $g(x, y(t))=0$. Let us consider the perturbed problem on this solution $y_{0}(t, x)$ :

$$
\begin{gathered}
\dot{\tilde{x}}=\varepsilon\left[f\left(t, \tilde{x}, y_{0}(t, \tilde{x}(t))\right)+A(\tilde{x}) \varphi(t, u)\right], \quad \tilde{x}(0)=x^{0}, \\
\tilde{J}[u]=\Phi(\tilde{x}(T)) \rightarrow \min ,
\end{gathered}
$$

We use one of the schemes averaging optimal control problems. Suppose that uniformly by $x$ there exists the limit:

$$
f_{0}(x)=\lim _{T \rightarrow \infty} \frac{1}{T} \int_{0}^{T} f\left(t, x, y_{0}(t, x)\right) d t .
$$


Let function $\varphi(t, u)$ is $2 \pi$-periodic by $t$. Now we will construct an admissible control set:

$$
V=\frac{1}{2 \pi} \int_{0}^{2 \pi} \varphi(t, U) d t .
$$

Let us consider the following averaged optimal control problem

$$
\begin{gathered}
\dot{z}=\varepsilon\left[f_{0}(z)+A(z) v\right], \quad z(0)=x^{0}, \quad v(t) \in V \subset \operatorname{conv}\left(R^{r}\right), \\
J_{0}[v]=\Phi(z(T)) \rightarrow \min
\end{gathered}
$$

for the problem $(21)-(23)$.

Establish the correspondence between the control function $u(t)$ of the problem $(21)-(23)$ and the control function $v(t)$ of the averaged problem $(28),(29)$.

Suppose that there exists an optimal control $u^{*}(t) \in U$ of problem $(21)-$ (23).

We can construct the control function $\bar{v}(t) \in V$ of optimal control problem (28), (29) using the following formulas:

$$
\bar{v}(t)=\left\{\bar{v}_{i}(t): \int_{2 \pi i}^{2 \pi(i+1)} \varphi\left(t, u^{*}(t)\right) d t=\int_{2 \pi i}^{2 \pi(i+1)} \bar{v}_{i}(t) d t\right\} .
$$

Now we suppose that there exists an optimal control $v^{*}(t) \in V$ of averaged problem (28), (29).

We can construct the control function $\bar{u}(t) \in U$ of optimal control problem (21) - (23) using the following formulas:

$$
\bar{u}(t)=\left\{\bar{u}_{i}(t): \int_{2 \pi i}^{2 \pi(i+1)} \varphi\left(t, \bar{u}_{i}(t)\right) d t=\int_{2 \pi i}^{2 \pi(i+1)} v^{*}(t) d t\right\},
$$

Note that the control function $\bar{u}(t) \in U$ constructing by algorithm above can be not unique.

Theorem 2. Suppose that in domain $\left\{t \geq 0, x \in D \subset R^{n}, y \in Q \subset R^{m}\right.$, $\left.u \in U \subset \operatorname{comp}\left(R^{r}\right)\right\}$ the following conditions hold:

I) $f(t, x, y), \varphi(t, u), A(x)$ are continuous functions on $t$, uniformly bounded by the constant $M$ and satisfy the Lipschitz condition with respect to $x$ and $y$, function $\varphi(t, u)$ is continuous on $u, 2 \pi$-periodic on $t$;

II) The function $\Phi(x)$ satisfies a Lipschitz condition with respect to $x$; 
III) The conditions I, II, IV and V of Theorem 1;

IV) The limit (26) exists uniformly with respect to $x$;

$V$ ) for any admissible control $v(t) \in V$ the corresponding trajectory $z(t)$ of the averaged system (28) is defined by $t \geq 0$, and with its $\rho$-neighborhood is in domain $D$.

Then, for any $\eta>0$ and for any $L>0$ there exists $\varepsilon_{0}(\eta, L)>0$ such that for all $\varepsilon \in\left(0, \varepsilon_{0}\right]$ and $t \in[0, T]$ the relations are fulfilled:

A) if exists an optimal solution $u^{*}(t), x^{*}(t), y^{*}(t), J\left[u^{*}(t)\right]$ of the problem (21) - (23), there an admissible control $\bar{v}(t) \in V$ will be found built with (30) and the corresponding trajectory $\bar{z}(t)$ of the problem (28), (29)and the folloving estimates hold:

$$
\begin{gathered}
\left\|x^{*}(t)-\bar{z}(t)\right\| \leq \eta, \\
\left|J\left[u^{*}(t)\right]-J_{0}[\bar{v}(t)]\right| \leq \eta .
\end{gathered}
$$

B) if $v^{*}(t), z^{*}(t), J_{0}\left[v^{*}(t)\right]$ is a solution of the averaged problem (28), (29), then an admissible control $\bar{u}(t) \in U$ exists, built by (31), and the corresponding trajectory $\bar{x}(t)$ of the system (21) - (23) satisfied the next estimates

$$
\begin{gathered}
\left\|z^{*}(t)-\bar{x}(t)\right\| \leq \eta, \\
\left|J_{0}\left[v^{*}(t)\right]-J[\bar{u}(t)]\right| \leq \eta .
\end{gathered}
$$

Proof. Let us consider the problem (21) - (23), for which exists an optimal solution $u^{*}(t), x^{*}(t), y^{*}(t), J\left[u^{*}(t)\right]$. For any admissible control functions $u(t) \in U$, including the optimal control $u^{*}(t)$, condition III) of the theorem provides for the systems (21), (22) and (24) satisfaction the conditions of Theorem 1.

Therefore, for any $\eta_{1}>0$ and for any $L>0$ there exists $\varepsilon_{1}\left(\eta_{1}, L\right)>0$ such that for all $\varepsilon \in\left(0, \varepsilon_{1}\right]$ and for all $t \in[0, T]$ the following inequality hold:

$$
\left\|x^{*}(t)-\tilde{x}(t)\right\| \leq \eta_{1} .
$$

Inequality (36) holds for each $t \in[0, T]$, so with the condition II) of the theorem we have that

$$
\begin{gathered}
\left|J\left[u^{*}(t)\right]-\tilde{J}\left[u^{*}(t)\right]\right|=\left|\Phi\left(x^{*}(T)\right)-\Phi(\tilde{x}(T))\right| \leq \\
\leq \gamma\left\|x^{*}(T)-\tilde{x}(T)\right\| \leq \gamma \eta_{1} .
\end{gathered}
$$


Now we consider the auxiliary problem for the problem (24), (25)

$$
\begin{gathered}
\dot{\tilde{z}}=\varepsilon\left[f_{0}(\tilde{z})+A(\tilde{z}) \varphi(t, u)\right], \quad \tilde{z}(0)=x^{0}, \\
\tilde{\tilde{J}}[u]=\Phi(\tilde{z}(T)) \rightarrow \min ,
\end{gathered}
$$

For any admissible control functions $u(t) \in U$, including those for the optimal control $u^{*}(t)$, the conditions I), III), IV), V) of the theorem ensure that the conditions of partial averaging theorem 2.4 [1] satisfied.

Therefore, for any $\eta_{2}>0$ and selected $L>0$ exists $\varepsilon_{2}\left(\eta_{2}, L\right)>0$ such that for all $\varepsilon \in\left(0, \varepsilon_{2}\right]$ and for all $t \in[0, T]$ the inequality holds

$$
\|\tilde{x}(t)-\tilde{z}(t)\| \leq \eta_{2}
$$

Moreover, it is fair

$$
\begin{gathered}
\left|\tilde{J}\left[u^{*}(t)\right]-\tilde{\tilde{J}}\left[u^{*}(t)\right]\right|=|\Phi(\tilde{x}(T))-\Phi(\tilde{z}(T))| \leq \\
\leq \gamma\|\tilde{x}(T)-\tilde{z}(T)\| \leq \gamma \eta_{2} .
\end{gathered}
$$

For the optimal control $u^{*}(t)$ of the problem $(21)-(23)$ we will construct control $\bar{v}(t) \in V$ for the averaged system (28), based on the algorithm (30). The system of differential equations (28) with the control $\bar{v}(t) \in V$ is partially averaged to the system of differential equations(38) with the control $u^{*}(t)$. Conditions I), III), IV), V) of the theorem ensure the fulfillment of the conditions of theorem $2.4[1]$.

Therefore, for the selected $L>0$ exist $\varepsilon_{3}(L)>0$ and $C_{1}(L)>0$ such that for all $\varepsilon \in\left(0, \varepsilon_{3}\right]$ and for all $t \in[0, T]$ the following inequation would be valid

$$
\|\tilde{z}(t)-\bar{z}(t)\| \leq C_{1} \varepsilon
$$

In addition, taking into consideration condition II) of the theorem we obtain

$$
\begin{gathered}
\left|\tilde{\tilde{J}}\left[u^{*}(t)\right]-J_{0}[\bar{v}(t)]\right|=|\Phi(\tilde{z}(T))-\Phi(\bar{z}(T))| \leq \\
\leq \gamma\|\tilde{z}(T)-\bar{z}(T)\| \leq \gamma C_{1} \varepsilon .
\end{gathered}
$$

Thus, if exists $u^{*}(t), x^{*}(t), y^{*}(t), J\left[u^{*}(t)\right]-$ the optimal solution of the problem $(21)-(23)$, we can construct an admissible control $\bar{v}(t) \in V$ of problem $(28)-(29)$, defined by the equation (30), and the corresponding trajectory $\bar{z}(t)$ of the averaged system (28) such that for any $\eta>0$ and $L>0$ 
exists $\varepsilon_{0}(\eta, L)>0$ such that for all $\varepsilon \in\left(0, \varepsilon_{0}\right]$ and fo all $t \in[0, T]$ the following inequations would be valid

$$
\begin{gathered}
\left\|x^{*}(t)-\bar{z}(t)\right\| \leq \\
\leq\left\|x^{*}(t)-\tilde{x}(t)\right\|+\|\tilde{x}(t)-\tilde{z}(t)\|+\|\tilde{z}(t)-\bar{z}(t)\| \leq \eta_{1}+\eta_{2}+C_{1} \varepsilon, \\
\left|J\left[u^{*}(t)\right]-J_{0}[\bar{v}(t)]\right| \leq \gamma \eta_{1}+\gamma \eta_{2}+\gamma C_{1} \varepsilon .
\end{gathered}
$$

Let choose $\varepsilon_{0}>0$ so that $\max \left\{\eta_{1}+\eta_{2}+C_{1} \varepsilon_{3}, \gamma\left(\eta_{1}+\eta_{2}+C_{1} \varepsilon_{3}\right)\right\} \leq \eta$, we will obtain the estimate (32), (33) of the theorem. The first part of the theorem is established.

Let us prove the second part of the theorem. A set of feasible controls $V$, constructed according to (27), when the condition 1) of theorem is fulfilled, would be convex and compact. Consequently, exists an optimal solution $v^{*}(t), z^{*}(t), J_{0}\left[v^{*}(t)\right]$ of the averaged problem (28), (29). On optimal control $v^{*}(t)$ and according to the algorithm (31) let us construct control $\bar{u}(t)$. Conditions I), III), IV), V) of the theorem ensure the fulfillment of the conditions of theorem 2.4 [1].

The system of differential equations (38) with the control $\bar{u}(t)$ is partially averaged towards the system of differential equations (28) with the control $v^{*}(t)$. Conditions I), III), IV), V) of the theorem and the construction (31) ensure the fulfillment of the conditions of the theorem 2.4 [1].

Therefore, for the selected $L>0$ one could find $\varepsilon_{4}(L)>0$ and $C_{2}(L)>0$ such that for all $\varepsilon \in\left(0, \varepsilon_{4}\right]$ and for all $t \in[0, T]$, the following inequations would be valid

$$
\begin{gathered}
\left\|z^{*}(t)-\tilde{z}(t)\right\| \leq C_{2} \varepsilon, \\
\left|J_{0}\left[v^{*}(t)\right]-\tilde{\tilde{J}}[\bar{v}(t)]\right|=\left|\Phi\left(z^{*}(T)\right)-\Phi(\tilde{z}(T))\right| \leq \\
\leq \gamma\left\|z^{*}(T)-\tilde{z}(T)\right\| \leq \gamma C_{2} \varepsilon .
\end{gathered}
$$

Then, the problem (38), (39) is partially averaged towards the perturbed problem (24), (25) and the conditions I), III), IV), V) of the theorem ensure the fulfillment of the conditions of theorem 2.4 [1] on the partial averaging.

Therefore, for any $\eta_{5}>0$ and selected $L>0$ exists $\varepsilon_{5}\left(\eta_{5}, L\right)>0$ such that for all $\varepsilon \in\left(0, \varepsilon_{5}\right]$ and for all $t \in[0, T]$, the following inequations would be valid

$$
\begin{gathered}
\|\tilde{z}(t)-\tilde{x}(t)\| \leq \eta_{5} . \\
|\tilde{\tilde{J}}[\bar{u}(t)]-\tilde{J}[\bar{u}(t)]|=|\Phi(\tilde{z}(T))-\Phi(\tilde{x}(T))| \leq \\
\leq \gamma\|\tilde{z}(T)-\tilde{x}(T)\| \leq \gamma \eta_{5} .
\end{gathered}
$$


However, for the perturbed problem (24), (25) and problem (21) - (23) on the same feasible controls $\bar{u}(t) \in U$ for any $\eta_{6}>0$ and selected $L>0$ there is such $\varepsilon_{6}\left(\eta_{6}, L\right)>0$ that for all $\varepsilon \in\left(0, \varepsilon_{6}\right]$ and $t \in[0, T]$, the following inequations would be valid

$$
\begin{gathered}
\|\tilde{x}(t)-\bar{x}(t)\| \leq \eta_{6} . \\
|\tilde{J}[\bar{u}(t)]-J[\bar{u}(t)]|=|\Phi(\tilde{x}(T))-\Phi(\bar{x}(T))| \leq \\
\leq \gamma\|\tilde{x}(T)-\bar{x}(T)\| \leq \gamma \eta_{6} .
\end{gathered}
$$

Thus, if exists $v^{*}(t), z^{*}(t), J_{0}\left[v^{*}(t)\right]$ - the optimal solution of the averaged problem (28), (29), we can construct a feasible control $\bar{u}(t) \in U$, defined by the equation (31), and the corresponding trajectory $\bar{x}(t)$ of the system $(21)$ such that for any $\eta>0$ and $L>0$ exists $\varepsilon_{0}(\eta, L)>0$ such that for all $\varepsilon \in\left(0, \varepsilon_{0}\right]$ and for all $t \in[0, T]$ the following inequations would be valid

$$
\begin{gathered}
\left\|z^{*}(t)-\bar{x}(t)\right\| \leq \\
\leq\left\|z^{*}(t)-\tilde{z}(t)\right\|+\|\tilde{z}(t)-\tilde{x}(t)\|+\|\tilde{x}(t)-\bar{x}(t)\| \leq C_{2} \varepsilon+\eta_{5}+\eta_{6}, \\
\left|J_{0}\left[v^{*}(t)\right]-J[\bar{u}(t)]\right| \leq \gamma C_{2} \varepsilon+\gamma \eta_{5}+\gamma \eta_{6} .
\end{gathered}
$$

Let choose $\varepsilon_{0}>0$ so that $\max \left\{C_{2} \varepsilon_{4}+\eta_{5}+\eta_{6}, \gamma\left(C_{2} \varepsilon_{4}+\eta_{5}+\eta_{6}\right)\right\} \leq \eta$, we will obtain assessments (34), (35) of the theorem, and the control construction $\bar{u}(t)$ is asymptotically optimal for the original optimal control problem $(21)-$ (23). The theorem is established.

\section{References}

[1] V.A. Plotnikov, The Averaging Method in Control Problems, Lybid, KievOdessa (1992), In Russian.

[2] A.B. Vasilieva, V.F. Butuzov, Asymptotic Expansions of Solutions of Singularly Perturbed Equations, Nauka, Moskow (1973), In Russian.

[3] I.A. Boitcova, Construction of asymptotic solutions of problem with fast and slow variables in a conditionally stable case, Vest. OGU. Fiz.-Mat. Nauki, 8, No. 2 (2003), 113-120, In Russian. 